\title{
New singularities in nonrelativistic coupled channel scattering. I. Second order
}

\author{
N. N. Khuri \\ Department of Physics, The Rockefeller University, New York, New York 10021 \\ Tai Tsun Wu \\ Gordon McKay Laboratory, Harvard University, Cambridge, Massachusetts 02138-2901 \\ and Theoretical Physics Division, CERN, CH-1211 Geneva 23, Switzerland
}

(Received 7 May 1997)

\begin{abstract}
New and surprising singularities are found in the forward scattering amplitude for nonrelativistic potential scattering with coupled channels. In the simplest case of two coupled channels, these singularities appear when the energy difference between the two channels is larger than the inverse range of the potential. They are similar to singularities recently discovered by one of us for potential scattering on $R^{3} \otimes S^{1}$.
\end{abstract}

[S0556-2821(97)03923-4]

PACS number(s): 03.80. $+\mathrm{r}, 03.65 . \mathrm{Nk}$

\section{INTRODUCTION}

For almost 40 years, it has been generally believed that both in quantum field theory [1] (QFT) as well as in nonrelativistic potential scattering [2-4], a common feature is the absence of singularities in the forward amplitudes on the physical sheet (except possibly for bound-state poles). This property is the main ingredient needed to obtain the forward dispersion relations which can be tested by experiment. The completion of the CERN Large Hadron Collider (LHC) will enable us to test the dispersion relations in an important new domain. The important fact here is that at LHC energies one is exploring the validity of local QFT at energy scales which have not been previously explored by quantum electrodynamics (QED). From the $(g-2)$ theory and experiment [5], one can put an upper bound on the existence of a "fundamental length" $L$, and get $L<(1 \mathrm{TeV})^{-1}$. Calculating $(g-2)$ in QED to tenth order will not improve this bound since at that level hadronic and electroweak contributions, which cannot be accurately calculated, will begin to make significant contributions to $(g-2)$. In all previous tests of the dispersion relations, QED had at a preceding time given us a better check on QFT. At LHC energies this will be different, and one will be exploring an explored shortdistance domain.

In this paper we will demonstrate that, in coupled-channel nonrelativistic potential scattering, new and surprising singularities (even simple poles) can appear in the forward amplitude on the physical sheet.

The old proofs of the forward dispersion relation in quantum field theory include, in addition to the general properties of causality and relativistic invariance, an additional, and always considered, technical assumption. For example, for a scattering $a+a \rightarrow a+a$, one has to exclude the possibility that $a+\bar{a} \rightarrow b+\bar{b}$ can happen with $m_{b} \ll m_{a}$. For the case of $\bar{p} p \rightarrow \pi \pi$, this ruins the $p p \rightarrow p p$ proof unless $\left(m_{\pi} / m_{p}\right)$ is much larger than it actually is. This has always been considered a technicality that can, somehow, be avoided by better methods. We shall show however that, insofar as potential scattering is a good guide, the problem could be much more serious.
Most of this paper deals with the case of two coupled channels where $T_{n m}\left(\vec{k}, \vec{k}^{\prime}\right)$ is a $2 \times 2$ matrix, and the potential $V_{m n}$ has Yukawa off-diagonal entries. We explicitly calculate $T_{11}(k)$ and $T_{22}(k)$ for the forward case in secondorder perturbation theory and show that, while $T_{11}^{(2)}(k)$ is analytic on the physical sheet as expected, $T_{22}^{(2)}(k)$ has a pole at $k=i\left(a^{2}+\mu^{2}\right) / 2 \mu$, where $a^{2}$ is the energy difference between the two channels, $E_{2}-E_{1}=a^{2}$, and $\mu^{-1}$ is the Yukawa range with $a^{2}>\mu^{2}$.

The pole in $T_{22}^{(2)}(k)$ on the physical sheet is unexpected. It is definitely not a bound state since it appears in perturbation theory. It is interesting to have a better understanding of these singularities and to look for physical systems where their presence may lead to detectable effects.

Our main task is to understand this new singularity better. It would be helpful to know if it appears in the full amplitude. This, so far, we have not achieved. The next best thing is to look at higher orders. It turns out that the next order where this same singularity could appear is the fourth order in perturbation. This is already a complicated problem, and we tackle it in an accompanying paper. It is found that the singularity appears there too and at the same point, $k=+i\left(a^{2}+\mu^{2}\right) / 2 \mu$.

A historical remark is now in order, namely what motivated us to consider this problem. As is often the case in physics, the path is quite indirect. More than two years ago, one of us studied the problem of nonrelativistic potential scattering on a space with an additional internal compact dimension [6], more specifically $R^{3} \otimes S^{1}$. The motivation for examining such a problem arose in the context of the proposed existence [7] of a new compact internal dimension, with radius $R$ where $R^{-1} \sim 1 \mathrm{TeV}$. The question is whether such a new compact dimension would lead to a violation of the forward dispersion relations, and hence could be detected experimentally. This led one of us to look at a simple welldefined model: nonrelativistic quantum mechanics on $R^{3} \otimes S^{1}$. The results were surprising. The analyticity properties [2-4] which are true for $R^{3}$ do not hold in $R^{3} \otimes S^{1}$. Indeed new poles appeared on the physical sheet in second- 
order perturbation theory whenever $R^{-1}>\mu$, where $\mu^{-1}$ is the range of the potential.

In Sec. II, we give a brief review of this $R^{3} \otimes S^{1}$ case. In the following section we show that the same result occurs when $S^{1}$ is replaced by $N$ discrete equally spaced points on a circle of radius $R$, i.e., $R^{3} \otimes Z_{N}$. The new poles are there for all $N>1$, including $N=2$ which is just a two-coupledchannel problem. Again the only requirement is that $1 / R>\mu$.

In Sec. IV, we define a simple two-channel problem, and proceed to calculate the second-order forward scattering amplitude. We show that, under similar conditions, the same pole appears in the forward amplitude.

\section{THE $R^{3} \otimes S^{1}$ CASE}

We sketch in this section the main result of [6].

The Schrödinger equation on $R^{3} \otimes S^{1}$, written in dimensionless form, is

$$
\left[\vec{\nabla}^{2}+\frac{1}{R^{2}} \frac{\partial^{2}}{\partial \phi^{2}}+K^{2}-V(r, \phi)\right] \Psi(\vec{r}, \phi)=0,
$$

where $\vec{r} \in R^{3}, R$ is the fixed radius of $S^{1}$, and $\phi$ is the angle on $S^{1}$. The potential $V(r, \phi)$ is taken to be periodic in $\phi$, i.e., $V(r, \phi)=V(r, \phi+2 \pi)$. One also assumes from the beginning that there are two scales, $1 / R>\mu$, where $\mu^{-1}$ is the range of the force in $R^{3}$. The normalized free solutions of Eq. (2.1) are

$$
\psi_{0}(\vec{x}, \phi)=\frac{1}{(2 \pi)^{2}} e^{i \vec{k} \cdot \vec{x}} e^{i n \phi}, \quad n=0, \pm 1, \pm 2, \ldots,
$$

and the total energy is $K^{2}=k^{2}+n^{2} / R^{2}$.

The free Green's function is given by

$$
\begin{aligned}
G_{0}\left(K ; \vec{x}, \phi ; \vec{x}^{\prime}, \phi^{\prime}\right)= & -\frac{1}{(2 \pi)^{4}} \\
& \times \sum_{n=-\infty}^{+\infty} \int d^{3} p \frac{e^{i \vec{p} \cdot\left(\vec{x}-\vec{x}^{\prime}\right)} e^{i n\left(\phi-\phi^{\prime}\right)}}{p^{2}+n^{2} / R^{2}-K^{2}-i \epsilon}
\end{aligned}
$$

The main surprising result of [6] is the appearance of a pole in the second-order calculation of $T_{n n}^{(2)}(K)$, the forward scattering amplitude for $n \geqslant 1$. It suffices to consider the simple potential

$$
V(r, \phi)=2 \frac{e^{-\mu r}}{r} \cos \phi
$$

A straightforward calculation then gives us

$$
\begin{aligned}
T_{n n}^{(2)}(K)= & -\left.\frac{1}{2 \pi^{2}} F_{1}\left(k ; a^{2}\right)\right|_{a^{2}=(2 n-1) / R^{2}} \\
& -\left.\frac{1}{2 \pi^{2}} F_{2}\left(k ; a^{2}\right)\right|_{a^{2}=(2 n+1) / R^{2}},
\end{aligned}
$$

where $F_{1}$ and $F_{2}$ are given by

$$
\begin{aligned}
& F_{1}\left(k ; a^{2}\right)=\int d^{3} p \frac{1}{\left[(\vec{p}-\vec{k})^{2}+\mu^{2}\right]^{2}\left[p^{2}-\left(k^{2}+a^{2}\right)-i \epsilon\right]}, \\
& F_{2}\left(k ; a^{2}\right)=\int d^{3} p \frac{1}{\left[(\vec{p}-\vec{k})^{2}+\mu^{2}\right]^{2}\left[p^{2}-\left(k^{2}-a^{2}\right)-i \epsilon\right]} .
\end{aligned}
$$

The above integrations were performed in [6]; see Eqs. (4.10) and (4.11). In particular, we have

$$
F_{1}\left(k ; a^{2}\right)=\frac{\pi^{2} i}{4 k \mu^{2}}\left[\frac{\sqrt{k^{2}+a^{2}}+k-i \mu}{k-i\left(a^{2}+\mu^{2}\right) / 2 \mu}+\frac{\sqrt{k^{2}+a^{2}}-k-i \mu}{k+i\left(a^{2}+\mu^{2}\right) / 2 \mu}\right] .
$$

Continuing the above expression into the region $\operatorname{Im} k \geqslant 0$, there is a pole at

$$
k=+i \frac{a^{2}+\mu^{2}}{2 \mu}
$$

The residue $\left(\sqrt{k^{2}+a^{2}}+k-i \mu\right)$ does not vanish at this pole. In addition to the above pole, $F_{1}\left(k ; a^{2}\right)$ has branch points at $k= \pm i a$. The branch cut is taken to join these two points since we know that $T_{n n}^{(2)}(K)$ is analytic for large enough $|k|$, Im $k>0$. Also, with $1 / R>\mu, a>\mu$ and, hence, $\left(a^{2}+\mu^{2}\right) / 2 \mu>\mu$, and the pole lies above the branch point.

Finally, both $F_{2}$ and $T_{00}^{(2)}$ have no unusual singularities.

\section{THE CASE $\boldsymbol{R}^{3} \otimes Z_{N}$}

In order to shed more light on the origin of the unusual pole found in [6] and reviewed in the preceding section, we consider a discrete version of the previous model. Physically, such a discrete model is also of more relevance to atomic and nuclear systems. Instead of $S^{1}$, we take a set of $N$ discrete points on a circle of radius $R$, i.e., $R^{3} \otimes Z_{N}$.

There are $N$ discrete internal states $n=0,1, \ldots, N-1$. The previous model corresponds to $N \rightarrow \infty$. Thus we have $\phi \rightarrow \phi_{j}, j=0, \ldots, N-1 ; \phi_{j}=2 \pi j / N$. If we define the shift operator $D: j \rightarrow j+1$, then we replace $\partial^{2} / \partial \phi^{2}$ by

$$
\frac{\partial^{2}}{\partial \phi^{2}} \rightarrow\left(\frac{N}{2 \pi}\right)^{2}\left(D-2+D^{-1}\right)
$$

After making the above replacement, the Schrödinger equation becomes

$$
\left[\nabla^{2}+\frac{1}{R^{2}}\left(\frac{N}{2 \pi}\right)^{2}\left(D-2+D^{-1}\right)+K^{2}-V(r, j)\right] \Psi(r, j)=0 .
$$

More explicitly, we have 


$$
\begin{aligned}
& {\left[\nabla^{2}-\frac{2}{R^{2}}\left(\frac{N}{2 \pi}\right)^{2}+K^{2}-V(r, j)\right] \Psi(r, j)} \\
& +\frac{1}{R^{2}}\left(\frac{N}{2 \pi}\right)^{2}[\Psi(r, j+1)+\Psi(r, j-1)]=0 .
\end{aligned}
$$

The free solution is now

$$
\psi_{0}\left(\vec{x}, \phi_{j}\right)=\left(\frac{1}{2 \pi}\right)^{2} e^{i \vec{k} \cdot \vec{x}} e^{i n \phi_{j}}, \quad \phi_{j}=\frac{2 \pi j}{N}
$$

The total energy of this state is

$$
\begin{aligned}
K^{2} & =k^{2}-\frac{1}{R^{2}}\left(\frac{N}{2 \pi}\right)^{2}\left[e^{i n(2 \pi / N)}-2+e^{-i n(2 \pi / N)}\right] \\
& =k^{2}+\frac{1}{R^{2}}\left(\frac{N}{\pi}\right)^{2} \sin ^{2} \frac{n \pi}{N} .
\end{aligned}
$$

Thus the replacement is

$$
\frac{n^{2}}{R^{2}} \rightarrow \frac{1}{R^{2}}\left(\frac{N}{\pi}\right)^{2} \sin ^{2} \frac{n \pi}{N}
$$

The Green's function is now

$$
G_{0}\left(K ; \vec{x}, j ; \vec{x}^{\prime}, j^{\prime}\right)=-\frac{1}{(2 \pi)^{4}} \sum_{n=0}^{N-1} \int d^{3} p \frac{e^{i \vec{p} \cdot\left(\vec{x}-\vec{x}^{\prime}\right)} e^{i n\left(j-j^{\prime}\right)}}{p^{2}+\left(1 / R^{2}\right)(N / \pi)^{2} \sin ^{2}(n \pi / N)-K^{2}-i \epsilon}
$$

From this point onward, the calculation of $T_{n n}^{(2)}$ is very similar to the preceding case. In $F_{1}, a^{2}$ is now given through the replacement (3.6), and since

$$
a^{2}=\frac{2 n-1}{R^{2}} \equiv \frac{n^{2}}{R^{2}}-\frac{(n-1)^{2}}{R^{2}},
$$

the new $a^{2}$ for $F_{1}$ is

$$
\begin{aligned}
a^{2} & =\frac{1}{R^{2}}\left(\frac{N}{\pi}\right)^{2}\left[\sin ^{2} \frac{\pi n}{N}-\sin ^{2} \frac{(n-1) \pi}{N}\right] \\
& =\frac{1}{R^{2}}\left(\frac{N}{\pi}\right)^{2} \sin \frac{\pi}{N} \sin \frac{(2 n-1) \pi}{N} .
\end{aligned}
$$

The final result for $n \geqslant 1$ is

$$
\begin{aligned}
& T_{n n}^{(2)}(K) \\
& \quad=-\left.\frac{1}{2 \pi^{2}} F_{1}\left(k ; a^{2}\right)\right|_{a^{2}=\left(1 / R^{2}\right)(N / \pi)^{2} \sin (\pi / N) \sin [(2 n-1) \pi / N]} \\
& \quad-\left.\frac{1}{2 \pi^{2}} F_{2}\left(k ; a^{2}\right)\right|_{a^{2}=\left(1 / R^{2}\right)(N / \pi)^{2} \sin (\pi / N) \sin [(2 n-1) \pi / N]} .
\end{aligned}
$$

Here, $F_{1}$ and $F_{2}$ are given by Eq. (2.6) and the result for $F_{1}$ explicitly given by Eq. (2.7). Again we have a pole in $T_{n n}^{(2)}$, $n \geqslant 1$, at

$$
k=+i \frac{a^{2}+\mu^{2}}{2 \mu}
$$

but with $a^{2}$ given in Eq. (3.9).

The only condition we need to satisfy is

$$
\frac{1}{R^{2}}\left(\frac{\pi}{N}\right)^{2} \sin \frac{\pi}{N} \sin \frac{(2 n-1) \pi}{N}>\mu^{2} .
$$

Next we expand the wave functions $\Psi(\vec{r}, j)$ in terms of the internal eigenstates of the internal momentum operator, $i^{-1} \partial / \partial \phi_{j}$, which are given by $(1 / \sqrt{N}) \exp (i 2 \pi n j / N)$ for internal state $n$. We define $\Phi_{n}(\vec{r})$ by

$$
\Psi(\vec{r}, j)=\frac{1}{\sqrt{N}} \sum_{n=0}^{N-1} \Phi_{n}(\vec{r}) e^{i 2 \pi j n / N}
$$

where now $\Phi_{n}(\vec{r})$ represents a wave function in a specific internal eigenstate $n$. Inverting the sum in Eq. (3.13) we get

$$
\Phi_{n}(\vec{r})=\frac{1}{\sqrt{N}} \sum_{j=0}^{N-1} \Psi(\vec{r}, j) e^{-i 2 \pi j n / N}
$$

Multiplying Eq. (3.3) by $(1 / \sqrt{N}) e^{i 2 \pi j n / N}$ and summing over $j$, we get

$$
\begin{array}{r}
{\left[\nabla^{2}+K^{2}-\frac{1}{R^{2}}\left(\frac{N}{\pi}\right)^{2} \sin ^{2} \frac{\pi n}{N}\right] \Phi_{n}(\vec{r})} \\
\quad-\sum_{n^{\prime}=0}^{N-1} U\left(r, n-n^{\prime}\right) \Phi_{n^{\prime}}(\vec{r})=0,
\end{array}
$$

where

$$
U(r, n) \equiv \frac{1}{N} \sum_{j=0}^{N-1} V(r, j) e^{i 2 \pi j n / N},
$$

and

$$
U(r, n)=U(r, n+N) .
$$

Finally, in [6] we used the explicit example where 


$$
V(r, \phi)=2 \frac{e^{-\mu r}}{r} \cos \phi
$$

We can replace this by

$$
V(r, j)=2 \frac{e^{-\mu r}}{r} \cos \frac{2 \pi j}{N}
$$

Using Eq. (3.17) this gives

$$
U(r, n)=\frac{e^{-\mu r}}{r}\left(\delta_{n, 1}+\delta_{n,-1}\right)
$$

The phenomenon of [6] is already present for $N=2$, and the Schrödinger equation in that case is

$$
\left(\begin{array}{cc}
\nabla^{2}+K^{2} & -\frac{e^{-\mu r}}{r} \\
-\frac{e^{-\mu r}}{r} & \nabla^{2}+K^{2}-\frac{1}{R^{2}}\left(\frac{2}{\pi}\right)^{2}
\end{array}\right)\left(\begin{array}{l}
\Phi_{1}(r) \\
\Phi_{2}(r)
\end{array}\right)=0 .
$$

This is just a $2 \times 2$ coupled-channel problem with $E_{1}=K^{2}, \quad E_{2}=k^{2}$, and $K^{2}=k^{2}+a^{2}, \quad$ and in this case $a^{2}=\left(1 / R^{2}\right)(2 / \pi)^{2}$.

\section{THE TWO-CHANNEL PROBLEM}

We consider the following two-channel Hamiltonian,

$$
H=\left(\begin{array}{cc}
-\nabla^{2} & V(r) \\
V(r) & -\nabla^{2}+a^{2}
\end{array}\right),
$$

where $a$ is real and fixed. The free eigenstates of $H$ and the internal state, with momentum $\vec{k}$, are

$$
\phi_{1}=\left(\begin{array}{c}
e^{i \vec{k} \cdot \vec{x}} \\
0
\end{array}\right), \quad \phi_{2}=\left(\begin{array}{c}
0 \\
e^{i \vec{k} \cdot \vec{x}}
\end{array}\right)
$$

with energies $E_{1}=k^{2}$ and $E_{2}=K^{2}=k^{2}+a^{2}$. The fact that no potential terms appear along the diagonal in Eq. (4.1) is not relevant to the rest of this paper. One could easily add $U_{11}(r)$ and $U_{22}(r)$ without affecting the main result.

For this problem there are two forward scattering amplitudes, $T_{11}(K)$ and $T_{22}(K)$, with $\sqrt{E}=K$.

To demonstrate that the phenomenon of [6] already exists in this simple problem, we will calculate explicitly $T_{22}^{(2)}(K)$ in second-order perturbation theory for the case

$$
V(r)=\frac{e^{-\mu r}}{r} .
$$

We will find later that $T_{11}(K)$ has no unusual singularities for Im $K>0$. The free Green's function associated with $H$ defined in Eq. (4.1) is, for energy $E=K^{2}$,

$$
G(K)=\left(\begin{array}{cc}
G_{0}(K) & 0 \\
0 & G_{0}(k)
\end{array}\right)
$$

where $\quad\left(H-K^{2}\right) G(K ; \vec{x}-\vec{y})=\delta^{3}(\vec{x}-\vec{y}) \quad$ and $\quad G_{0}(q)$ $=-(1 / 4 \pi) e^{i q|x-y|} /|\vec{x}-\vec{y}|$.

For forward scattering from an initial state

$$
\left(\begin{array}{c}
0 \\
e^{i \vec{k} \cdot \vec{x}}
\end{array}\right)
$$

to the same outgoing state we have

$$
T_{22}^{(2)}(K)=\frac{1}{\pi} \int d^{3} x \int d^{3} x^{\prime} e^{-i \vec{k} \cdot \vec{x}} V(x) G_{0}\left(K ;\left|\vec{x}-\vec{x}^{\prime}\right|\right) V\left(x^{\prime}\right) e^{i \vec{k} \cdot \overrightarrow{x^{\prime}}}
$$

The off-diagonal nature of $V$ makes $G_{0}(K)=G_{11}$ appear in $T_{22}^{(2)}$ and not $G_{0}(k)=G_{22}$. This is the main feature leading to the new singularity.

Going to momentum space, we obtain

$$
T_{22}^{(2)}=-\frac{1}{2 \pi^{2}} F(K),
$$

where $F(K)$ is given by

$$
F(K)=\int d^{3} p \frac{1}{\left(p^{2}+\mu\right)^{2}\left[(\vec{p}+\vec{k})^{2}-K^{2}-i \epsilon\right]} .
$$

This is represented by Fig. 1. With a change of variables, this is identical to the expression for $F_{1}$ in Eq. (2.6), with $K^{2}=k^{2}+a^{2}$. For $k$ and $a$ real, this integral was calculated in [6], and is the same as Eq. (2.7), i.e.,

$$
F(K)=\frac{\pi^{2} i}{4 k \mu^{2}}\left[\frac{\sqrt{k^{2}+a^{2}}+k-i \mu}{k-i\left(a^{2}+\mu^{2}\right) / 2 \mu}+\frac{\sqrt{k^{2}+a^{2}}-k-i \mu}{k+i\left(a^{2}+\mu^{2}\right) / 2 \mu}\right] .
$$

With $a^{2}>\mu^{2}$ we can now continue $F$ into the region Im $k>0$. Again there is the pole at $k=+i\left[\left(a^{2}+\mu^{2}\right) / 2 \mu\right]$. The residue of this pole does not vanish since 


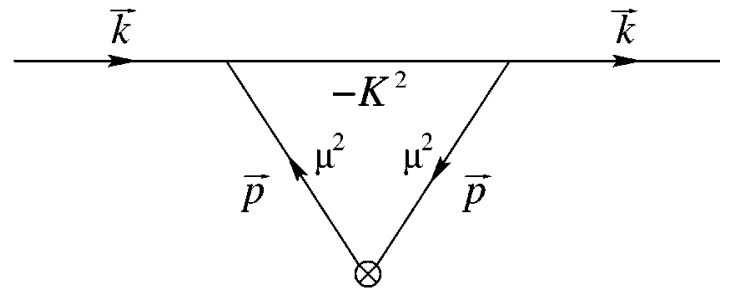

FIG. 1. Feynman diagram for the second-order amplitude. The propagator for the horizontal internal line has imaginary mass $=-i K=-i \sqrt{k^{2}+a^{2}}$.

$$
\left.\sqrt{k^{2}+a^{2}}\right|_{\text {pole }}=\sqrt{-\frac{\left(a^{2}+\mu^{2}\right)}{4 \mu^{2}}+a^{2}}=i \frac{\left(a^{2}-\mu^{2}\right)}{2 \mu}
$$

and

$$
\left[\sqrt{k^{2}+a^{2}}+k-i \mu\right]_{\mathrm{pole}}=i\left(\frac{a^{2}}{\mu}-\mu\right) \neq 0 .
$$

$F$ also has two branch points at $k= \pm i a$. These must be joined by a branch cut, since we know a priori that $F$ is analytic for large $|k|$, Im $k>0$. Finally, with $a^{2}>\mu^{2}$,

$$
\frac{a^{2}+\mu^{2}}{2 \mu}>a,
$$

and the pole is above the branch point at $k=+i a$. Using the variable $K, E=K^{2}$, we get

$$
K_{\text {pole }}^{2}=-\frac{\left(a^{2}+\mu^{2}\right)^{2}}{4 \mu^{2}}+a^{2}=\frac{-\left(a^{2}-\mu^{2}\right)^{2}}{4 \mu^{2}}
$$

and

$$
K_{\text {pole }}=+i \frac{\left(a^{2}-\mu^{2}\right)}{2 \mu} .
$$

For $a^{2}>\mu^{2}$, the RHS of Eq. (4.13) is positive and the pole is on the physical energy sheet. The calculation of $T_{11}^{(2)}$ is straightforward and leads to

$$
T_{11}^{(2)}=\frac{-\pi^{2}}{4 k \mu}\left[1+\frac{-i \mu}{k+i \mu / 2}\right] .
$$

The only pole is at $k=-i \mu / 2$, on the unphysical sheet.

Finally, we should stress the obvious fact that only for a pure Yukawa $V(r)$ do we get a simple pole. If we choose $V(r)=\int_{\mu_{0}}^{\infty} C(\mu) e^{-\mu r} d \mu$, i.e., a superposition of Yukawa, then the pole becomes a branch cut but still on the physical sheet.

\section{REMARKS}

The results of this paper present us with two problems, one mathematical and the other physical.

The mathematical question concerns this new singularity and whether it appears in the full amplitude, not just in second order. This has not yet been accomplished. However, in the accompanying paper we study the fourth-order twochannel problem. We find that a singularity appears at the same point $k=+i\left(a^{2}+\mu^{2}\right) / 2 \mu$, and its strength is the same as a pole. The position of the singularity remains unchanged in fourth order. This suggests strongly that the full forward amplitude indeed has a singularity located at $k=+i\left(a^{2}+\mu^{2}\right) / 2 \mu$.

The second question relates to the relevance of our result to physics. Is it just a mathematical oddity, or does there exist real physical models where our result manifests itself? The nuclear physicists deal normally with the situation where $a^{2}<\mu^{2}$, not $a^{2}>\mu^{2}$. In other words, they deal with coupled channels whose energy difference is small compared to the mass of the pion. The splitting of the levels is produced by the same forces and cannot be too large. A more promising approach is to look for a coupled-channel problem where the channel splitting is generated by an external factor. For example, in atomic physics, one could look for a situation where the splitting is enhanced by a strong magnetic field while the potentials are essentially unchanged by the field. There also could be such examples in condensed matter physics. At present we have no concrete examples, but we are continuing our search.

In closing, we make two additional comments.

The first relates to the importance of perturbation theory as a guide. In quantum field theory it is foolhardy to attempt to prove an analyticity property which is known to be untrue in low order perturbation theory. In fact we know of no example where the full amplitude has a larger domain of analyticity than the perturbative amplitudes. In potential scattering this fact is even more far reaching. The general proofs of forward dispersion relations in [2-4] all proceed to first establish analyticity and asymptotic properties for the full Green's function of the Schrödinger equation under very general conditions. From these the analyticity of the forward amplitude follows. The established properties of the full Green's function are manifestly true for the free Green's function. Since the free Green's function gives the secondorder amplitude, any unusual singularity in second order must be taken very seriously. It will be extremely hard to circumvent such singularities in general.

The second comment concerns the relation of our new singularities to a physical phenomenon already discussed earlier in this section. At present we do not believe that the effect of these new singularities can be seen in any high energy particle physics experiment. However, as we stressed earlier, in atomic physics or condensed matter physics, where ordinary quantum mechanics applies, it is still possible to find a physical system where our results are relevant. We continue to search for such a system, and we hope others will also do the same.

\section{ACKNOWLEDGMENTS}

We thank Torleif Ericsson and André Martin for helpful discussions. We would also like to thank the Theory Division at CERN for its kind hospitality. This work was supported in part by the U.S. Department of Energy under Grant No. DE-FG02-91ER40651, Task B, and under Grant No. DE-FG02-84ER40158. 
[1] For a review, see H. Lehmann, Nuovo Cimento Suppl. 153, 154 (1959).

[2] N. N. Khuri, Phys. Rev. 107, 1148 (1957).

[3] A. Grossmann and T. T. Wu, J. Math. Phys. 2, 710 (1961).

[4] W. Hunziker, Helv. Phys. Acta 34, 593 (1961).
[5] T. Kinoshita, in Quantum Electrodynamics edited by T. Kinoshita (World Scientific, Singapore, 1990), p. 471.

[6] N. N. Khuri, Ann. Phys. (N.Y.) 242, 332 (1995).

[7] I. Antoniadis, Phys. Lett. B 246, 377 (1990); also I. Antoniadis, C. Munoz, and M. Quiros, Nucl. Phys. B397, 515 (1993). 